\title{
Upper Limb Prosthesis Evaluation in Amman-Jordan
}

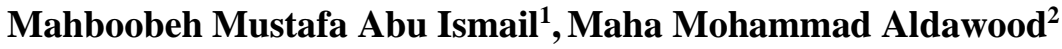 \\ ${ }^{1}$ Royal Medical Services, Amman, Jordan \\ ${ }^{2}$ University of Jordan, Amman, Jordan
}

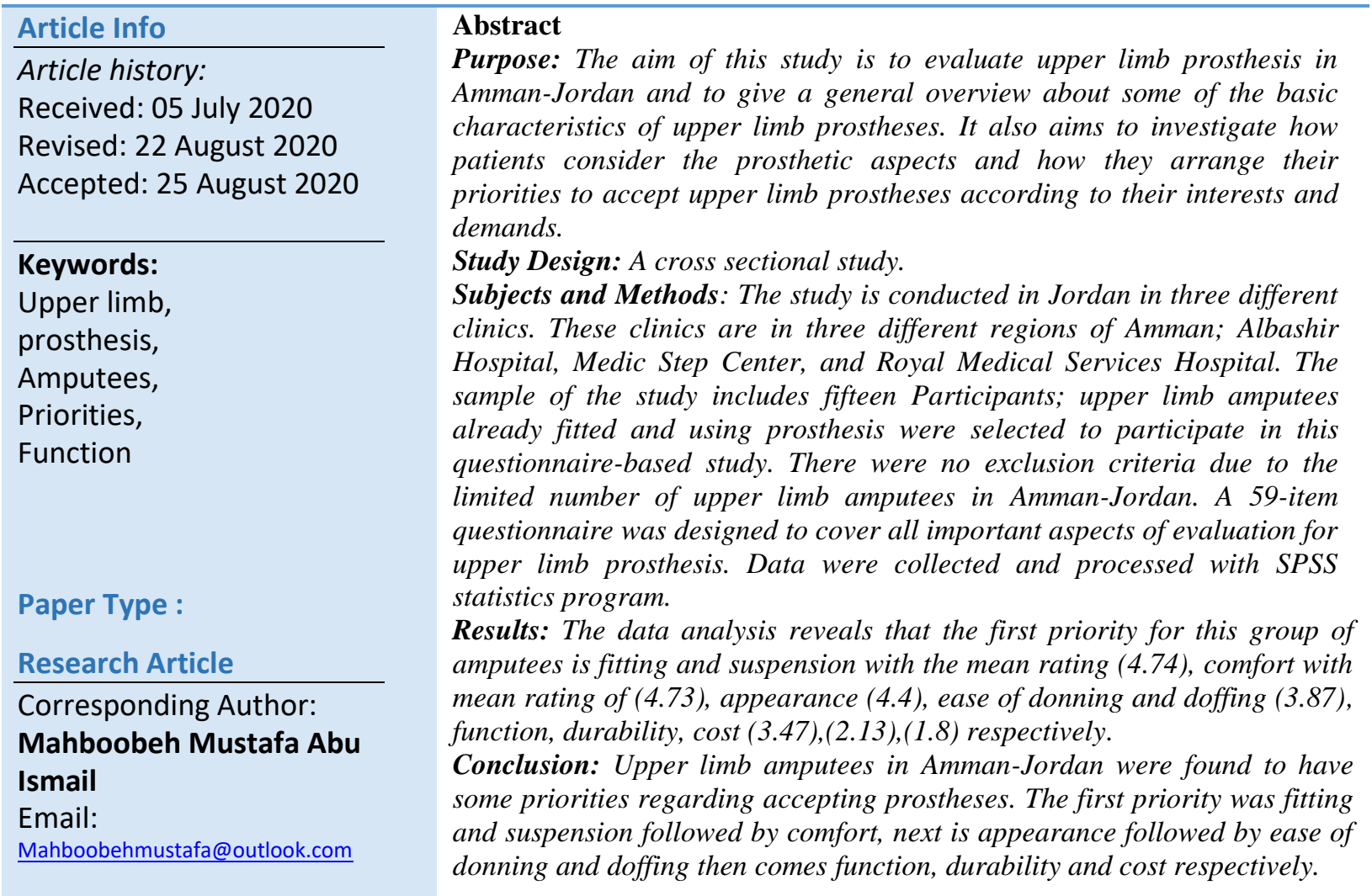

\section{Introduction}

Amputation is the removal of a limb by trauma, illness, or surgery to control pain or disease progression, such as malignancy or gangrene. In cases of inborn amputation, it is called congenital; where fetal limbs have been cut off (amputee-coalition.org. 2012). An amputation might occur to upper or lower limb's segments or sometimes to both. Amputations are divided into minor amputations which cause minor effect or disability, such as amputation to the very distal segments of the upper or lower limbs, and major amputations which cause severe disability and cause the loss of major joints of the body such as the knee or the elbow joints or above.

The most common causes for upper limb amputation are trauma in which the limb cannot be saved, congenital in cases of focomelia or polydactyly, infections, tumor $^{1}$ or for

\footnotetext{
${ }^{1}$ See Tawake undergoes surgery to remove finger, Australian Rugby Union.

ISSN: 2709-0159 (Print)

Copyright (c) 2020, Journal of Scientific Research in Medical and Biological Sciences (JSRMBS), Under 
legal punishment ${ }^{2}$. While in hospital and after release, a physical therapist helps with some exercises to strengthen the residual limb and to maintain the range of motion for upper limb amputees. Starting the therapy immediately after amputation gives the patient a great psychological advantage. The therapists are also responsible for training the amputees to use the prosthesis.

For the prosthesis to compensate well for the anatomical segment loss; many features should be present in it. Assessment of these features along with other criteria is considered to be evaluation of the prosthesis. Knowing that design priorities reflect patient's needs and goals for using the prosthesis and vary depending on prosthesis type and consumer age (Biddiss et al., 2007), evaluation of upper limb prosthesis is assessed in terms of type of prosthesis, functional units, fitting, comfort, appearance, weight, durability, ease of use, sensory feedback and cost (Dalsey et al., 1989). Among these parameters, the type of the prosthesis differs according to the patient's needs, condition and monetary status (Parker, et al., 2006).

Upper limb prosthetics are either passive or active. Passive prosthetics (cosmetic) have no movable parts, while active prosthetics may be body powered or electrically powered. Hybrid prosthesis is a combination of the two previous systems. Myoelectric prosthesis utilizes the electrical signals from the voluntary movements of residual limb's muscles to operate the terminal device after a series of signal processing (Dalsey et al., 1989, Parker, et al., 2006).

In general, as found by Roeschlein et al. (2009), factors related to successful upper limb prosthetic use were not completely based on the actual successful rehabilitation services provided; rather, they were more related to the perception about the monetary value of the prosthesis and the number of complicating factors which are; high school education, employment, fast return to work and finally self and social acceptance of the prosthesis (Roeschlein et al., 2009). Moreover, Biddiss et al., (2007) reported that weight of the prosthesis was noted to be the first priority a consumer would ask for, and then comes cost to be within the first five priorities for any patient (Biddiss et al., 2007).

Given all this, priorities might vary depending on the type of the prosthesis. Some of these variations were reported by Biddiss et al. (2007). Regarding passive or cosmetic prosthesis, one of the most important priorities is the life-like look of the prosthesis (Biddiss et al., 2007). Regarding body-powered prosthesis patients, major priorities are comfortability of the harness system used, wrist rotation, grip control and strength (Biddiss et al., 2007). For electrically-powered prosthesis, glove durability, poor proficiency and lack of sensation were the most important aspects the patient cared for (Biddiss et al., 2007).

Concerning ease and difficulty with use, Dudkiewicz et al. (2003) stated that no significant effect of amputation level except for trans-wrist amputation with $100 \%$ use of prosthesis (Dudkiewicz et al., 2003p.16). Moreover, Biddiss et al. (2009) in his review about upper limb prosthesis use and abandonment in the duration of 25 years identified that the prosthesis rejection percentages were $23 \%$ and $26 \%$ of electrically-powered and body-powered prosthesis respectively (Biddiss et al., 2009, p.250). Recently, Lindner et al.(2010) stated that

\footnotetext{
${ }^{2}$ See : UNHCR article on Amputation as a punishment in Iran ISSN: 2709-0159 (Print)

Copyright (C) 2020, Journal of Scientific Research in Medical and Biological Sciences (JSRMBS), Under the license CC BY- 4.0
} 
using a mixture of parameters in evaluation would give a better indication of the patient and the prosthesis (Lindner et al., 2010).

All previous studies describe the evaluation and characteristics of upper limb prostheses in different regions of the world. Unfortunately, there are no previous studies about the characteristics of upper limb prosthesis in Jordan or even in the Middle East. Therefore, this study was conducted in 2019 with an aim to provide a general assessment of the upper limb prostheses available in Amman, Jordan. This research is the first of a kind in country and thus it serves as a base for researches in the upper limb prosthetics field in Jordan. This research paper is an attempt to illustrate the characteristics of upper limb prosthesis in Amman-Jordan and to provide a general overview of upper limb amputee's states, concerns, demands and priorities of different prosthetic aspects using a special designed questionnaire for the research purposes including the criteria that were found to be important regarding upper limb amputees and prostheses in Amman-Jordan.

\section{Methodology and Procedures}

The sample of the study includes fifteen upper limb amputees who are already fitted and using prosthesis. These amputees were invited to participate in this questionnaire-based study. They were admitted and received medical treatment Albashir Hospital, Medic Step Centre, and Royal Medical Services.

The inclusion criteria were upper limb amputation at any level, good cognitive abilities (to be able to answer the questions), and the exclusion criteria were minor amputations (digit amputations) and patients who are not wearing prosthesis. More importantly, due to the limited numbers of upper limb amputees in Amman-Jordan, no significant exclusion criteria were carried out through the study.

A number of fifty four aspects that were deemed significant to be included in the questionnaire were identified and categorized from very general to very specific. They are categorized into relevant groups, such as personal information of the patient, amputation information, health of the residual limb, range of motion of remaining joints, types and evaluation of the prosthesis, and the main priorities in the prosthesis according to each patient. Then, we developed a scale according to the significance of the aspects. After that, the statements were typed, arranged and reviewed and used as a questionnaire to evaluate the amputees, allowing the patient to assess some of the parameters based on his/her case. The second part of the questionnaire allows the examiner to assess the cases individually with reference to international parameters.

A 4-page questionnaire is given to each participant and it is explained to him/her. The questions are answered mostly by the amputee with help of the researchers and data are recorded by the examiner, missing answers were looked for immediately by examiner in order to reduce its probability.

In terms of ethics, consent was gained to see the patients at each of the clinics. All the subjects were asked for permission at the beginning. Data collection took place in private rooms and away from others to maintain confidentiality. Patient's information was kept confidential, no names or descriptions of patients were recorded. 
For Data analysis, SPSS statistical program version 20 was used. The method of analysis includes mean and percentage. The data received in response to the questionnaire is presented in tables and figures. In addition, the results of the study are compared with other previous studies.

\section{Results and Discussion}

In this study, fifteen patients participated with the mean age of (26.1 15.36$)$, all with unilateral upper limb amputations. The participants consisted of five females (33.3\%) and ten male patients $(66.7 \%)$. The majority of upper limb amputees were transradial amputees $(53.3 \%)$ followed by shoulder disarticulation and transhumeral amputation (13.3\% for each). Furthermore, a small number of the patients were of wrist articulation (20.0\%).

Table 1: Type of Amputation

\begin{tabular}{|l|c|}
\hline Type of Amputation & Percentage \\
\hline Transradial Amputees & $53.3 \%$ \\
\hline Shoulder Disarticulation & $13.3 \%$ \\
\hline Transhumeral Amputation & $13.3 \%$ \\
\hline Wrist Articulation & $20.0 \%$ \\
\hline
\end{tabular}

The most common cause of amputation in the participant group was congenital amputation (46.7\%). More specifically, trauma comes in the second place (40\%) followed by tumour and other causes $6.7 \%$ for each. By the time of data collection, $40.0 \%$ of participants did not use their prostheses due to different reasons. The most common reason for not wearing prostheses is bad appearance $(77.8 \%)$ as reported by the participants. The other reasons were weight and pain as $11.11 \%$ for each.

Table 2: Common Causes of Amputation

\begin{tabular}{|l|c|}
\hline \multicolumn{1}{|c|}{ Causes } & Percentage \\
\hline Congenital Amputation & $46.7 \%$ \\
\hline Trauma & $40 \%$ \\
\hline Tumour & $6.7 \%$ \\
\hline Other Causes & $6.7 \%$ \\
\hline
\end{tabular}

Regarding prosthetic priorities, the aspect that was prioritized first by the majority of the amputees was fitting and suspension with a mean rate of 4.74 on a scale of 5 . This was followed by comfort with mean rate of 4.73. In addition, appearance comes in the third place with mean rate of 4.4 followed by ease of donning and doffing (mean rate of 3.87). The least prioritized aspects as viewed by the participants were function, durability and cost (3.47), (2.13) and (1.8) respectively. One patient reported that he would like to ignore appearance to increase comfort $(6.67 \%)$, and four patients reported that they would ignore appearance to increase function (26.66\%). On the other hand, ten patients reported that they would ignore function to increase appearance $(66.67 \%)$.

The data analysis reveals that most of the amputees prioritize appearance. It seems that they are more concerned about how others perceive them. The psychological aspect of the amputation is reflected in their preference of appearance to any other properties of the ISSN: 2709-0159 (Print)

Copyright (C) 2020, Journal of Scientific Research in Medical and Biological Sciences (JSRMBS), Under the license CC BY- 4.0 
prosthesis. Since this study is limited to studying the priorities of prosthesis aspects, further research examining the psychological as well as social aspects would be useful. Any future research on this aspect would explain why amputees prefer to ignore function in order to have good appearance.

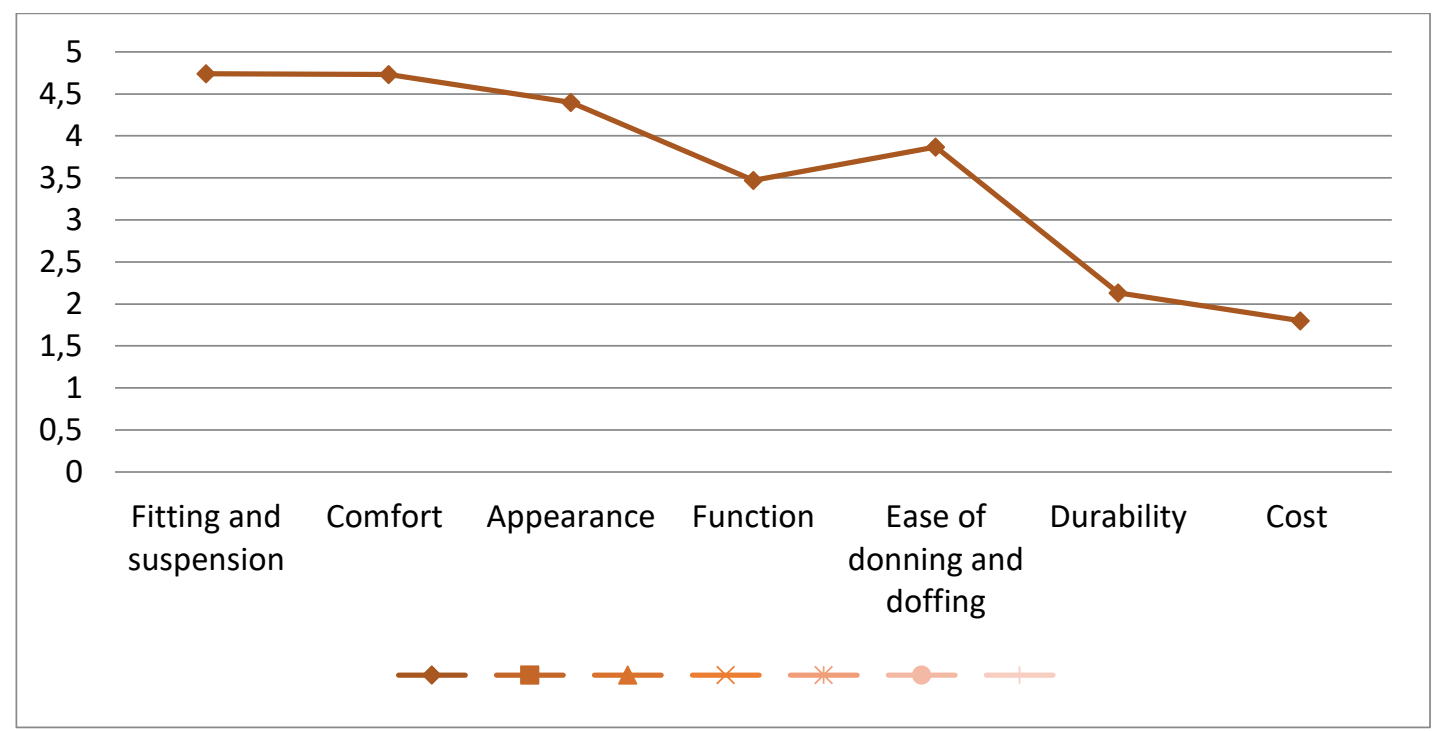

Figure 1: Prosthetic Priorities in Five-Point Scale

Based on the results of data analysis, fitting and suspension was the first priority for the amputees to ask for, which is inconsistent with what was published by Biddiss et al. (2007) reporting that weight of the prosthesis was noted to be the first priority a consumer would ask for. Furthermore, comfort was found to be the second priority of upper limb amputees in Amman-Jordan, since that comfort comes from good fitting and good suspension. This finding leads to the same previous finding, contradicting what Biddiss et al. (2007) reported about weight of the prosthesis to be the first priority for amputees.

Appearance of the prostheses is desired to be life-like regarding alignment, colour, shape and details. Based on the statistical analysis, amputees care too much about appearance. This concern about appearance caused (31.1\%) of the amputees to reject their prostheses due to bad appearance. Ease of donning and doffing of the prosthesis was reported to be a mediumlevel priority for the participants in this study. This can be explained by the fact that only a small number of the participants are body-powered prosthesis patients (20.0\%). In addition, as shown in figure 1, function was one of the last priorities for the participant since that all amputees were using cosmetic prostheses which have no actual function rather than compensating for the shape of the lost limb.

As presented in figure 1, durability and cost of the prosthesis were not that important to the group of participants because they were managed and fitted mainly at centres benefiting from military insurance or international relief agencies services. More specifically, $(6.67 \%)$ of the participants (one patient) preferred comfort over appearance due to the young age of the patient and the prominent bones in the residual limb. Furthermore, 26.66\% of the participants (four patients) favoured function over appearance. This can be attributed to their use of bodyISSN: 2709-0159 (Print)

Copyright (C) 2020, Journal of Scientific Research in Medical and Biological Sciences (JSRMBS), Under the license CC BY- 4.0 
powered prostheses. More importantly, $66.67 \%$ of the participants (ten patients) preferred appearance rather than function because they were using cosmetic prostheses.

Concerning ease and difficulty with use, it is stated that no significant effect of amputation level except for trans-wrist amputation with 100\% use of prosthesis (Dudkiewicz et al., 2003). The data analysis reveals that fitting and suspension was the first priority of amputees to ask for. It is inconsistent with what was published in other countries, reporting that weight of the prosthesis was noted to be the first priority a consumer would ask for. Comfort was also found to be the second priority. Almost all amputees aim to wear prostheses and want to feel comfortable wearing the prostheses.

\section{Conclusion and Suggestion}

The present study concludes that the upper limb amputees in Amman-Jordan were found to have some priorities regarding accepting prostheses. The first priority according to the participants was fitting and suspension followed by comfort. Compared to other studies, appearance is ranked third in terms of priority. It was followed by ease of donning and doffing, and then comes function, durability, and cost respectively.

Although some patients preferred comfort, some patients favoured appearance over function of the prosthesis and some other patients considered appearance to be the most important among all aspects. This variance in preference indicates that choosing the type of prosthesis depends on several medical, socio-economic, and personal factors. The present study has certain limitations. The number of participants in the study was small due to the limited number of upper limb amputees in Amman-Jordan. Articles in the field that are published in the literature do not cover all aspects of upper limb evaluation. In addition, no full access was available to many of the good articles. Hence, further research is needed to evaluate the cases of more patients and to make the study more valid in terms of sample number that is included.

\section{Conflict of Interest}

The authors of the article declare no conflict of interest.

\section{Funding:}

The authors received no funding for conducting this study. All costs associated with carrying out this research study were borne by the authors.

\section{References}

Amputee coalition factsheet, amputee-coalition.org. 2012-07-23. Retrieved 2013-04-22from https://www.amputee-coalition.org/resources/nevada-2/

Australian Rugby Union (2006-10-17) "Tawake undergoes surgery to remove finger" sportsAustralia.com retrieved 2013-04-22

Biddiss, E., Beaton, D., \& Chau, T. (2007). Consumer design priorities for upper limb prosthetics. Disability and rehabilitation: Assistive technology, 2(6), 346-357.

Biddiss, E. A., \& Chau, T. T. (2009). Upper limb prosthesis use and abandonment: a survey

ISSN: 2709-0159 (Print)

Copyright (C 2020, Journal of Scientific Research in Medical and Biological Sciences (JSRMBS), Under 
of the last 25 years. Prosthetics and orthotics international, 31(3), 236-257.

Dalsey, R., Gomez, W., Seitz Jr, W. H., Dick, H. M., Hutnick, G., \& Akdeniz, R. (1989). Myoelectric prosthetic replacement in the upper-extremity amputee. Orthopaedic Review, 18(6), 697-702.

Dudkiewicz, I., Gabrielov, R., Seiv-Ner, I., Zelig, G., \& Heim, M. (2004). Evaluation of prosthetic usage in upper limb amputees. Disability and rehabilitation, 26(1), 60-63.

Lindner, H. Y., Nätterlund, B. S., \& Hermansson, L. M. N. (2010). Upper limb prosthetic outcome measures: review and content comparison based on International Classification of Functioning, Disability and Health. Prosthetics and orthotics international, 34(2), 109-128.

Parker, P., Englehart, K., \& Hudgins, B. (2006). Myoelectric signal processing for control of powered limb prostheses. Journal of electromyography and kinesiology, 16(6), 541548.

Roeschlein, R. A., \& Domholdt, E. (2009). Factors related to successful upper extremity prosthetic use. Prosthetics and orthotics international, 13(1), 14-18.

United Nations High Commissioner for Refugees (2008-01-07). "UNHCR article on Amputation as a punishment in Iran" unhcr.org retrieved 2013-04-22 